\title{
Étude des obstacles et facilitateurs à la réintégration au travail de femmes atteintes du cancer du sein - 14/05/18 \\ Doi : 10.1016/j.admp.2018.03.118
}

\author{
Marianne Pelseneer ${ }^{1,3, *} \sqrt{ }$, Marina Milicevic ${ }^{2,3}$, Philippe Mairiaux $^{3}$, Anne-Cécile Willems ${ }^{1}$ \\ 1 CESI, Liège, Belgique \\ 2 Attentia, Liège, Belgique \\ 3 Université de Liège, Liège, Belgique
}

*Auteur correspondant.

\section{Résumé}

\section{Contexte}

Actuellement en Belgique, une femme sur neuf développe un cancer du sein avant l'âge de 75 ans. En 2015, 55 \% de ces femmes avaient moins de 65 ans et étaient donc en âge de travailler. La façon dont la femme réintègre son activité de travail reste cependant relativement méconnue des soignants. Cette étude a pour objectif d'explorer les dimensions essentielles du processus de retour au travail après cancer du sein en croisant les points de vue des patientes, et de leurs employeurs et collègues de travail, d'identifier les obstacles et les facilitateurs de la reprise du travail et de préciser le rôle que pourrait jouer le médecin du travail dans ce contexte.

\section{Méthodologie}

L'étude de nature principalement qualitative a comporté l'interview, après la reprise du travail, de 17 femmes ayant eu un cancer du sein, de 13 de leurs responsables hiérarchiques directs et de 10 de leurs collègues. Des données quantitatives ont été récoltées via un questionnaire comportant des questions explorant diverses dimensions comme l'estime de soi et l'état de fatigue.

\section{Résultats}

«Trois regards» ont été croisés et montrent des facteurs influençant de manière positive ou négative la reprise du travail, du point de vue des travailleuses (ex : + : temps partiel médical ; : fatigue), des employeurs (ex : + : avertissement de la reprise assez tôt ; - : reprise sans aménagement horaire) et des collègues (ex : + : contact gardé avec l'entreprise ; - : difficulté dans la relation d'aide lors de la reprise). Certains de ces facteurs sont communs aux 3 regards comme le maintien de contacts réguliers pendant l'arrêt de travail qui favorise la reprise soit propre à chaque regard. Lorsque les travailleuses sont vues en visite de pré-reprise, le médecin du travail valide ou aide à mettre en place les modalités pratiques de la reprise du travail.

\section{Conclusions}

Dans l'échantillon étudié, l'envie de reprendre le travail des femmes ayant eu un cancer du sein est souvent plus forte que les difficultés engendrées par la reprise et par leur travail. Mais pour préparer cette reprise, elles ne savent pas vers qui se tourner et elles connaissent peu la visite de pré-reprise ainsi que les aides que le médecin du travail et le médecin-conseil pourraient leur apporter. Le responsable hiérarchique direct est un interlocuteur prioritaire pour la travailleuse et joue un rôle important dans l'organisation de la reprise du travail. Il ressort également que la communication est primordiale entre la travailleuse, son responsable hiérarchique ainsi que ses collègues, afin que chacun puisse expliquer ses difficultés à l'autre et que la reprise du travail se passe dans les meilleures conditions possibles.

\section{Remerciements}

Financement du Fonds Scientifique CESI.

Le texte complet de cet article est disponible en PDF.

Mots clés : Cancer du sein, Retour au travail, Perspectives des travailleuses, Perspectives des employeurs, Perspectives des collègues 